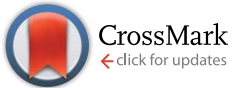

Cite this: RSC Adv., 2015, 5, 32078

Received 14th February 2015 Accepted 30th March 2015

DOI: $10.1039 / c 5 r a 02877 a$

www.rsc.org/advances

\section{A critical review on in situ reduction of graphene oxide during preparation of conducting polymeric nanocomposites}

\begin{abstract}
Prasanna Kumar S Mural, $\uparrow^{a}$ Maya Sharma, $\uparrow^{a}$ Giridhar Madras ${ }^{b}$ and Suryasarathi Bose*c
Graphene oxide (GO), prepared by chemical oxidation of graphite, serves as a building block for developing polymeric nanocomposites. However, their application in electrical conductivity is limited by the fact that the oxygen sites on GO trap electrons and impede charge transport. Conducting nanocomposites can be developed by reducing GO. Various strategies have been adopted to either reduce GO ex situ, before the composite preparation, or in situ during the development of the nanocomposites. The current state of research on in situ reduction of GO during the preparation of conducting polymeric nanocomposites is discussed in this review. The mechanism and the efficiency of reduction is discussed with respect to various strategies employed during the preparation of the nanocomposite, the type of polymer used, and the processing conditions employed, etc. Its overall effect on the electrical conductivity of the nanocomposites is also discussed and the future outlook in this area is presented.
\end{abstract}

\section{Introduction}

Graphite's lamellar structure is made up of individual layers that are held together by van der Waals forces and are considered to be independent entities (graphene). These independent entities are known to possess unique electronic and mechanical properties. Some of the remarkable electronic and mechanical properties of graphene reported are $\sim 200000 \mathrm{~cm}^{2} \mathrm{~V}^{-1} \mathrm{~s}^{-1}$ of charge carrier mobility, high Young's modulus of $\sim 1100 \mathrm{GPa}$ and extraordinary fracture strength of $125 \mathrm{GPa}$. Apart from high electrical conductivity, they possess very high specific surface area of $2630 \mathrm{~m}^{2} \mathrm{~g}^{-1}$ and interesting transport phenomena such as the quantum Hall effect. ${ }^{1,2}$ Besides flexibility in properties, mono layer graphene sheets offers myriad possibilities of chemical modification and functionalization. ${ }^{1,3}$ Thus, graphene can be potentially used as energy-storage materials, polymer composites and sensors etc. Yan et al. ${ }^{4}$ reported that graphene and few-layer graphene can be utilized for thermal management of advanced electronics wherein graphene acts as heat spreaders in transistors. Luo et al., ${ }^{5}$ summarized various graphene based applications in energy storage such as solar cells, lithium ion secondary batteries and supercapacitors. In a recent article, Mittal et al., ${ }^{6}$ reported that graphene exhibits restacking

\footnotetext{
${ }^{a}$ Center for Nano Science and Engineering, Indian Institute of Science, Bangalore-560012, India

${ }^{b}$ Department of Chemical Engineering, Indian Institute of Science, Bangalore-560012, India

${ }^{c}$ Department of Materials Engineering, Indian Institute of Science, Bangalore-560012, India.E-mail:sbose@materials.iisc.ernet.in

$\dagger$ Both PKS. Mural and M. Sharma contributed equally to this work.
}

of sheets due van der Waals forces and strong $\pi-\pi$ stacking effects. The interaction between graphene sheets and polymer matrix plays a vital role in achieving high electrical percolation. Graphene sheets can also act as a cationic initiator for polymerization of polystyrene (PS) and poly(styrene-isoprene). ${ }^{7}$ Graphene polymer composites can be used for tissue engineering applications such as osteogenesis in $3 \mathrm{D}$ scaffolds, ${ }^{8,9}$ selective gas/vapor sensors ${ }^{10}$ and in removal of heavy metal/pathogenic bacteria from aqueous media. ${ }^{11-14} \mathrm{GO}$ based composites showed a significant surface enhanced Raman scattering (SERS), ${ }^{15}$ which can be useful in developing ultrasensitive SERS-based immunosensing platforms. ${ }^{16-18}$

Graphite to individual 2D graphene conversion is widely carried out by chemical oxidation of graphite layer. The formed oxides serve as a precursor for cost-effective and mass production of graphene-based materials. ${ }^{2}$ These exfoliated layers of graphite sheets with oxygen moieties are referred to as graphene oxide (GO). GO offers flexibility in the large scale production of graphene based nanocomposite. ${ }^{19} \mathrm{GO}$ is widely synthesized via Brodie, ${ }^{20}$ Staudenmaier ${ }^{21}$ and Hummers methods. ${ }^{17,22}$ All these methods focus on the oxidation of individual layers using strong acids and oxidants for the introduction of oxide groups such as epoxy, hydroxyl or carboxyl. ${ }^{17,23}$ This oxidation results in the disruption of $\mathrm{sp}^{2}$ bonding. In addition, these oxygen sites trap electrons that results in electrically insulating materials. High electrical conductivity can be achieved by effective removal of oxygen species. Various strategies that are reported in the literature for restoring electrical conductivity are chemical reduction, thermally reduction, microwave assisted reduction, ${ }^{24}$ photocatalysis ${ }^{2,17,25,26}$ and in situ reduction of GO during processing. Apart from these techniques, some literature exist that 
utilized UV treatment for further reduction of GO. ${ }^{27}$ There are plenty of reviews available on the techniques mentioned above. ${ }^{2}$ However, the state of research on in situ reduction of GO, during the preparation of the nanocomposite, has not received much attention although the field is gaining significant interest among the scientific community.

Given the brevity of this review, we focus here mainly on the in situ reduction of GO during the preparation of conducting nanocomposites. The in situ reduction of GO is carried out mainly during in situ polymerization or during melt processing. ${ }^{28}$ The following section will highlight the current state of research on in situ reduction of GO to design and develop conducting polymeric nanocomposites from GO as the starting material.

\section{In situ polymerization and simultaneous reduction of GO}

The in situ polymerization involves polymerization of polar or hydrophilic monomers that can interact and intercalate into the stack of GO sheets. This results in exfoliation and isolation of GO sheets. To accomplish this, GO is initially dispersed in a polar solvent by ultrasonication. The dispersed GO sheets are then mixed with monomers and subsequently in situ polymerized at high temperature and under inert atmosphere. The in situ polymerization at high temperature ensures proper dispersion and reduction of GO. Hence, the obtained reduced GO composite shows 3-4 orders of higher conductivity compared to GO based composites. This strategy was adopted by Liu et al..$^{29}$ in which simultaneous dispersion and thermo-reduction of GO occurs during in situ melt polycondensation reaction. GO was initially dispersed in ethylene glycol and then the composites were prepared via in situ polymerization of terephthalic acid (PTA) (Fig. 1). Further, it was demonstrated that polyester chains were successfully grafted onto GO sheets during polymerization, accompanied by the thermo-reduction from GO to reduced graphene oxide. The reported increase in conductivity of the composite is $c a$. $0.56 \mathrm{~S} \mathrm{~m}^{-1}$ in striking contrast to GO based composites $\left(7 \times 10^{-4} \mathrm{~S} \mathrm{~m}^{-1}\right)$. They concluded that the chemical bonding between the polymer chain and GO is favorable for the improvement of interfacial interactions in the composites. In a recent study, Jin et al. ${ }^{30}$ adopted similar strategy to graft PBS (poly(butylene succinate)) chains onto graphene oxide during in situ polymerization, which is accompanied by the thermoreduction from GO to graphene. In an another recent work, ${ }^{31}$ an one-step approach was utilized to reduce and functionalize graphene oxide (GO) during the in situ polymerization of phenol and formaldehyde. The in situ reduction of graphene oxide provides homogenous dispersion of graphene into polymer matrix and additionally improves the electrical and thermal conductivity in the composite. ${ }^{32-36}$

\section{In situ reduction of GO during compositing process}

The in situ reduction of GO during compositing process involves dispersion of GO followed by thermal reduction resulting in a composite with monolayer reduced GO. This strategy offers a simple fabrication procedure that prevents restacking and aggregation of reduced GO sheets in a given polymer matrix. Fig. 2 illustrates the method of in situ reduction during compositing process.

Recently, Tang et al. ${ }^{37}$ explored this strategy for poly(vinylidene fluoride) PVDF based GO composites, wherein they showed wellisolated reduced GO sheets in PVDF. Initially, they dispersed GO in DMF to yield highly exfoliated GO followed by mixing with PVDF. Thus, obtained PVDF/GO composites were then hot pressed at $200{ }^{\circ} \mathrm{C}$ for $2 \mathrm{~h}$. Hot pressing leads to in situ reduction of GO that is evident from the change in color from brown to black.

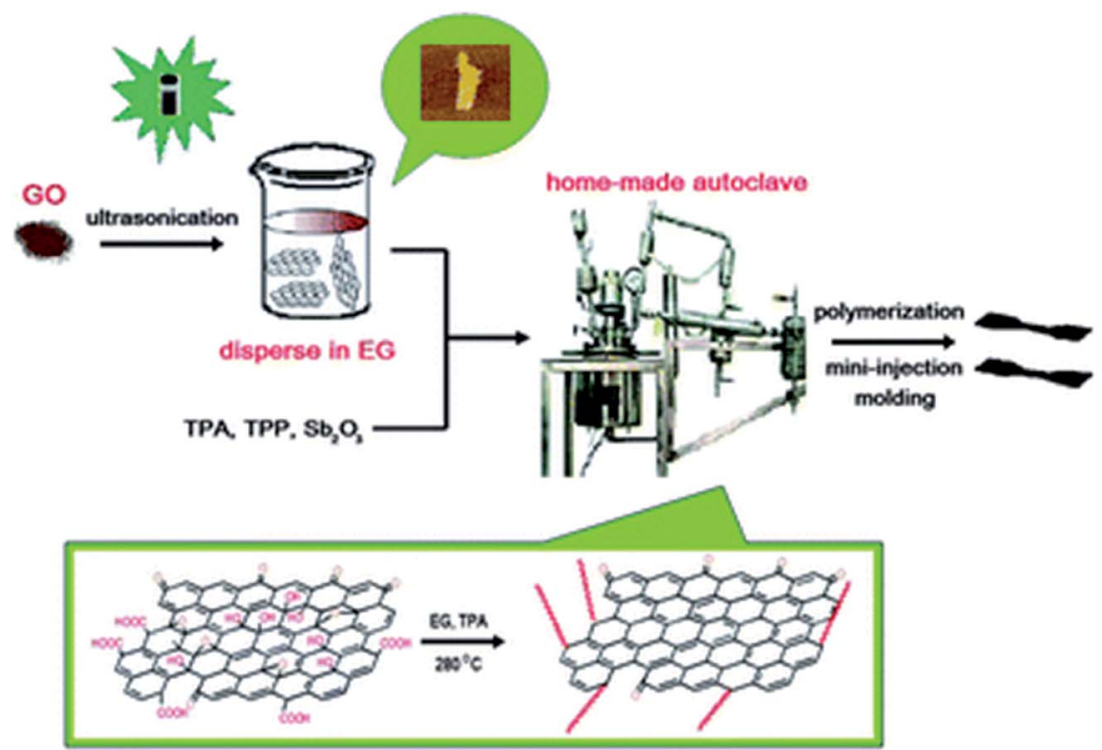

Fig. 1 The synthesis of reduced polymer composite via in situ melt polycondensation (Liu et al. ${ }^{29}$ reproduced by the permission of The Royal Society of Chemistry). 


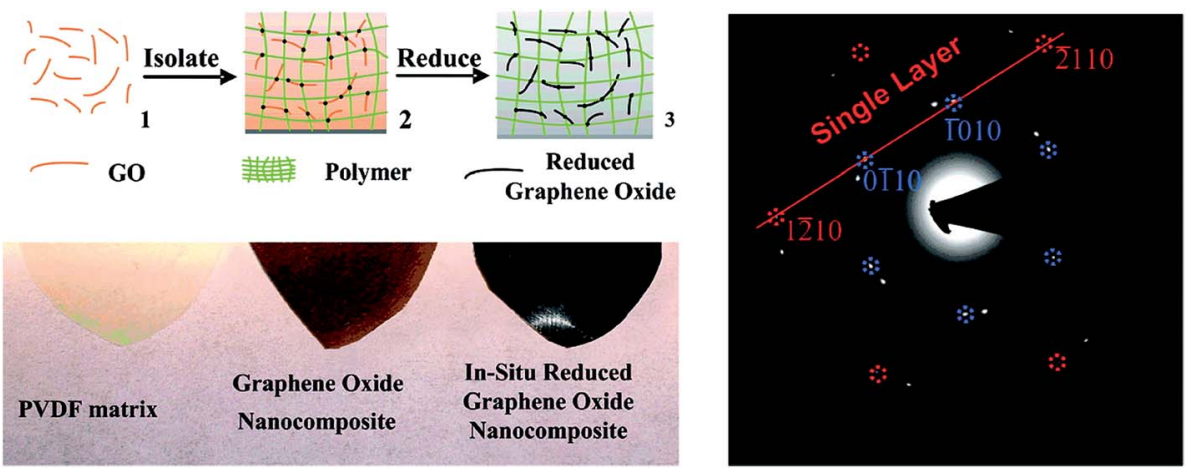

Fig. 2 In situ reduction during compositing process (Tang et al. ${ }^{37}$ reproduced by permission of American Chemical Society Copyright ( 2012, American Chemical Society).

This color change is attributed to the efficient removal of oxygen functional groups and partially restoring the graphitic structure. From Fig. 2, it is evident that PVDF/GO composite was initially brown suggesting uniform dispersion of GO in PVDF. However, after reduction, the films turned black. Restoring graphitic structure can lead to $\pi$-conjugation, which renders electrical conductivity in the composites. Further, they demonstrated a three-order increase in conductivity with respect to GO composites at a relatively low fraction of 0.16 vol\% of reduced GO. Due to immobilization of GO, thin layer of sheets percolate into composites and lead to low percolation threshold. The single layer of reduced GO was further confirmed by TEM (as shown in Fig. 2) and suggested that polymer with higher thermal stability could even enhance the conductivity by complete reduction of GO to graphene.

In another study, ${ }^{38} \mathrm{PVDF} / \mathrm{GO}$ composites were prepared by solution mixing in DMF. The resultant composite was coagulated with water. The obtained PVDF/GO mixture was then hot pressed at $200{ }^{\circ} \mathrm{C}$ at $50 \mathrm{MPa}$ into sheets of about $0.4 \mathrm{~mm}$ thickness. After hot pressing, the color of the composite changed from grey to black suggesting thermal reduction of GO into reduced GO. These sheets were used as master batch for the preparation of PVDF/GO composites using a melt compounder. They demonstrated a significant improvement in conductivity of the composite i.e. ca. $3 \times 10^{-7} \mathrm{~S} \mathrm{~cm}^{-1}$ at a loading of 0.17 vol\% of reduced GO. Further, at 1 vol\% of reduced GO, a conductivity of $2 \times 10^{-3} \mathrm{~S} \mathrm{~cm}^{-1}$ was obtained and the change in conductivity with processing temperature was studied. The processing temperature affects the percolation threshold of TRG. For instance, PVDF-TRG composite processed (hot pressed) at $200{ }^{\circ} \mathrm{C}$ exhibited a percolation threshold of $0.12 \pm 0.02 \mathrm{vol} \%$ (as shown in Fig. 3) in contrast to the composite processed at 190 and $210{ }^{\circ} \mathrm{C}$ which showed similar percolation thresholds of $0.17 \pm 0.02$ vol\% and $0.17 \pm 0.01$ vol\%, respectively (Table 1 ).

The reduction of GO in situ during compression molding was also studied by Ding-Xiang et $a l .{ }^{39}$ wherein they distributed GO on the surface of ultra-high molecular weight poly(ethylene) (UHMWPE) particles followed by compression molding. It is evident that GO sheets are distributed at the interface of UHMWPE particles and further reduction of GO was assisted by

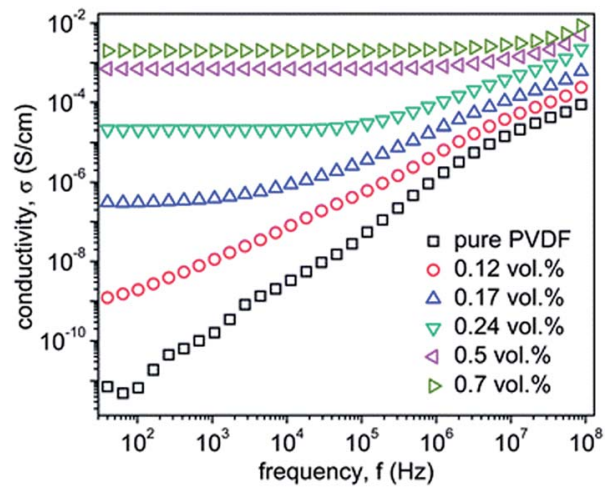

Fig. 3 Conductivity plot of PVDF with various vol\% of TRG-PVDF (He et al. ${ }^{38}$ reproduced by the permission of The Royal Society of Chemistry).

Table 1 Critical percolation threshold and electrical conductivity of reduced GO-PVDF composites hot-pressed at different temperature $^{38}$ (reproduced by the permission of The Royal Society of Chemistry)

\begin{tabular}{llc}
\hline Temperature $\left({ }^{\circ} \mathrm{C}\right)$ & $\begin{array}{l}\text { Critical percolation } \\
\left(P_{\mathrm{c}}\right)(\mathrm{vol} \%)\end{array}$ & $\begin{array}{l}\text { Conductivity } \\
\left(\sigma_{\mathrm{c}}\right)\left(\mathrm{S} \mathrm{cm}^{-1}\right)\end{array}$ \\
\hline 190 & $0.17 \pm 0.02$ & $75.29 \pm 16.23$ \\
200 & $0.12 \pm 0.02$ & $1496 \pm 136.38$ \\
210 & $0.17 \pm 0.02$ & $573.74 \pm 63.54$ \\
\hline
\end{tabular}

hot pressing at $280{ }^{\circ} \mathrm{C}$ and $10 \mathrm{MPa}$ pressure for $30 \mathrm{~min}$. Hot pressing ensured reduction of GO to reduced GO in situ. The distribution of reduced GO sheets at the interface yielded a low percolation threshold of 0.66 vol\% in UHMWPE and an electrical conductivity of $c a .3 .4 \mathrm{~S} \mathrm{~m}^{-1}$.

Yan et $a .^{40}$ utilized segregated architectures to distribute reduced GO at the interface of polymer granules rather than distributing in volume in the matrix. These segregated architectures lead to reduction of percolation threshold hence resulting in an increase in electrical conductivity. They utilized polystyrene (PS) of different particle size and observed PS with higher particle size to yield lower percolation threshold. They 

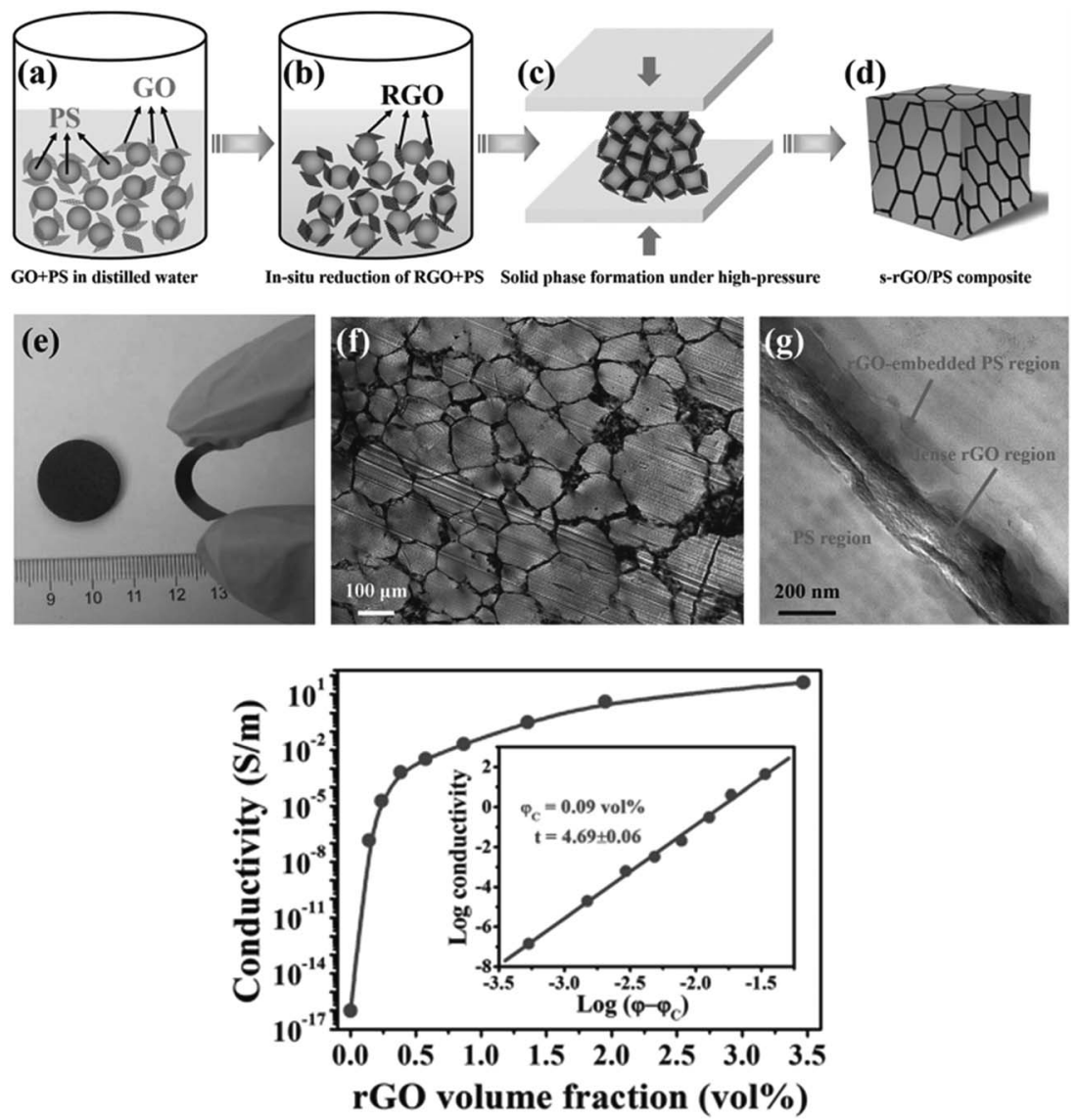

Fig. 4 Schematic of in situ reduction of GO in PS and its composite preparation with optical, TEM micrograph of rGO layers at interface of PS and Conductivity with varying in situ reduced GO in PS (Yan et al. ${ }^{40}$ reproduced by permission of John Wiley and Sons, Copyright @ 2014 Wiley Ltd. All rights reserved).

reported percolation threshold of 0.09 vol\% in PS and conductivity of order of $c a \cdot 10^{-2} \mathrm{~S} \mathrm{~m}^{-1}$ at $\sim 1$ vol\% of reduced GO (Fig. 4).

In another study by Shen et $a l .{ }^{41} \mathrm{GO}$ was mixed with poly(vinyl pyrrolidone) (PVP) and glucose (which acts as a reducing agent). GO-PVP and GO-glucose was then mixed with poly(lactic acid) (PLA) in DMF. The resultant solution containing GO-PVP and GO-glucose was then coagulated using methanol. The formed precipitate was dried, transferred to a mold and hot pressed at $210{ }^{\circ} \mathrm{C}$. This process results in the reduction of GO facilitated by both thermal and chemical processes. The resultant composite with 1.25 vol\% of GO-glucose showed a significant high conductivity of $2.2 \mathrm{~S} \mathrm{~m}^{-1}$ in striking contrast to neat PLA $\left(10^{-15} \mathrm{~S} \mathrm{~m}^{-1}\right)$. The high electrical conductivity was attributed to the improved exfoliation and dispersion of reduced GO glucose further facilitating in the formation of interconnected conducting network within the PLA matrix. Fig. 5 shows the electrical conductivity as a function of particle concentration for different PLA composites.

Zhu et $a .^{42}$ reported reduction of GO by dispersing in propylene carbonate (PC) at $\mathrm{pH} 3$ by bath sonication. The obtained PC solution was heat treated at $150{ }^{\circ} \mathrm{C}$ for $12 \mathrm{~h}$ resulting in reduced GO. Composite samples were dried in vacuum at $80{ }^{\circ} \mathrm{C}$, and resulted in a conductivity of 2100 and
$1800 \mathrm{~S} \mathrm{~m}^{-1}$ for the samples, which were heat treated at 150 and $200{ }^{\circ} \mathrm{C}$, respectively. Further, vacuum annealing at $250{ }^{\circ} \mathrm{C}$ for $12 \mathrm{~h}$ resulted in a conductivity of $5230 \mathrm{~S} \mathrm{~m}^{-1}$ and $2640 \mathrm{~S} \mathrm{~m}^{-1}$ for the samples which were subjected to heat treatment at 150 and $200{ }^{\circ} \mathrm{C}$, respectively. In this work, they also discussed the dispersion of GO in the PC matrix, and observed that the dispersion of GO in PC may not be due to electrostatic repulsion

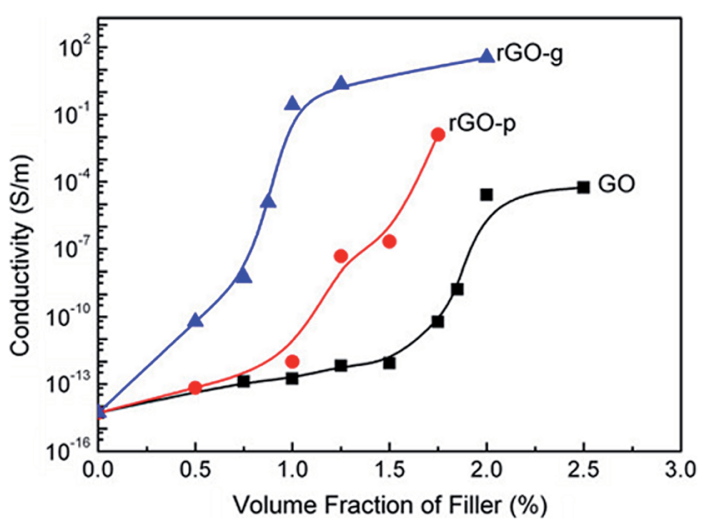

Fig. 5 Plots of electrical conductivity versus filler loading for PLA composites (Shen et al. ${ }^{41}$ reproduced by permission of Elsevier, Copyright @ 2012 Elsevier Ltd.). 


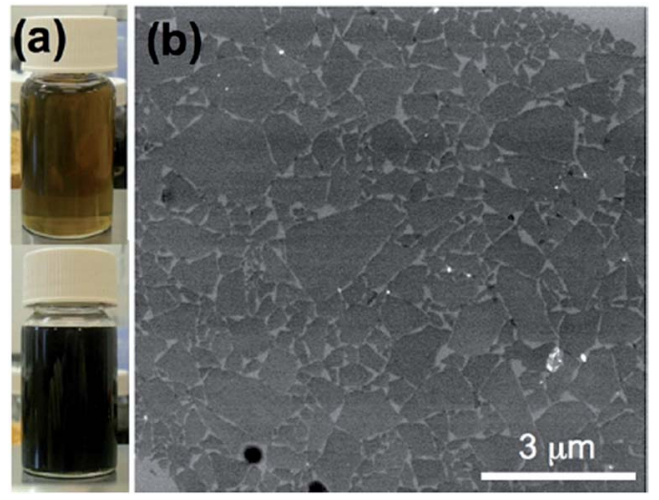

Fig. 6 (a) Optical images of a graphene oxide suspension in PC (top) before and (bottom) after heating at $150{ }^{\circ} \mathrm{C}$ for $12 \mathrm{~h}$. (b) SEM image of graphene oxide platelets deposited on a Si substrate (Zhu et al. ${ }^{42}$ reproduced by permission of American Chemical Society Copyright $(\odot$ 2010, American Chemical Society).

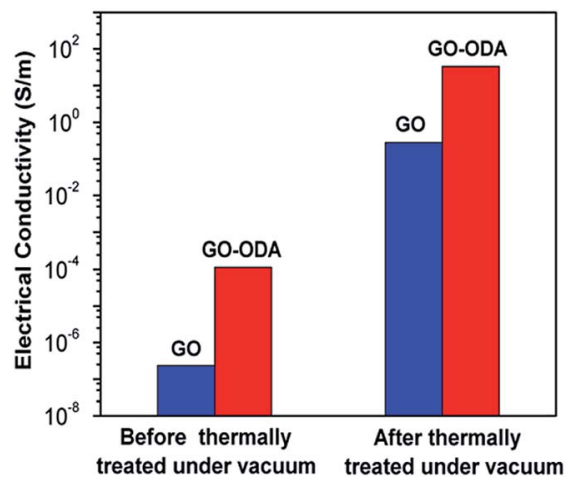

Fig. 7 The electrical conductivities of GO and GO-ODA before and after the thermal treatment ( $\mathrm{Li}$ et al. ${ }^{43}$ reproduced by permission of Elsevier, Copyright (C) 2011 Elsevier Ltd.).

but can be due to the high dipole moment of PC. Fig. 6 shows the state of dispersion of GO in PC as well as in the solvent.

In a recent study, in situ reduction of GO was reported during the compositing process in the presence of surface functionalized octadecylamine (ODA). ${ }^{43}$ The presence of long octadecyl chain resulted in the hydrophobicity in GO and also facilitated in its efficient reduction. Further, by hot pressing at $210{ }^{\circ} \mathrm{C}$ in the presence of PS matrix, electrically conducting samples were obtained. Thermal reduction in presence of PS resulted in a sharp transition from insulator to conductor. The GO-ODA showed 2 orders of higher electrical conductivity when subjected to thermal treatment as displayed in Fig. 7. Hence, it can be concluded that surface modification of GO along with thermal reduction may assist in both improved dispersion and simultaneous increase in electrical conductivity.

Another strategy commonly used to reduce GO is by changing polymer chemistry. The latter can play an important role in assisting thermal reduction of GO during hot pressing. Glover $e$ e $a l .{ }^{44}$ studied the in situ reduction of GO in poly(vinyl pyrrolidone), poly(vinyl acetate), and poly(vinyl pyrrolidone/ vinyl acetate) and they demonstrated that the chemical architecture exhibited by these polymer had strong influence on the extent of thermal reduction. They compared time/temperature relationship for GO reduction in air and in dimethylformamide under the same temperature conditions. The efficiency of reduction was reported based on the change in the $\mathrm{C} / \mathrm{O}$ (carbon/ oxygen) ratio (see Table 2). They showed that reduction of GO depends on time/temperature history and also on the polymer chemistry. They reported an increase in electrical conductivity by 2 orders in all the composites.

\section{Mechanism of in situ reduction: synergistic effects from functionalization and heat}

The thermal gravimetric analysis (TGA) of GO is characterized by three transitions which can be attributed to vaporization of hydroxyl group $\left(<100{ }^{\circ} \mathrm{C}\right)$ and loss of carbonyl group as $\mathrm{CO}$ or $\mathrm{CO}_{2}$ at $120-150{ }^{\circ} \mathrm{C}$ and $200-260^{\circ} \mathrm{C}$. From TGA of GO, it is clear that temperature above $200^{\circ} \mathrm{C}$ is critical for reduction of oxygen moieties on the GO surface and hence a processing temperature above $200^{\circ} \mathrm{C}$ will result in efficient reduction of GO. It is equally important to select a polymer that degrades well above $200^{\circ} \mathrm{C}$. Henceforth, optimum processing time, the thermal stability of

Table $2 \mathrm{C} / \mathrm{O}$ ratio of $\mathrm{GO}$ as a function of reduction time and temperature (Glover et al. ${ }^{44}$ reproduced by permission of American Chemical Society Copyright ( 2011 , American Chemical Society)

\begin{tabular}{|c|c|c|c|c|c|}
\hline Temperature $\left({ }^{\circ} \mathrm{C}\right)$ & Exposure time & Medium & $\mathrm{C}$ content $(\%)$ & O content $(\%)$ & Atomic $\mathrm{C} / \mathrm{O}$ ratio \\
\hline 25 & - & - & 56 & 39 & 1.9 \\
\hline 55 & 4 months & Water & 63 & 27 & 3.2 \\
\hline 150 & $10 \mathrm{~min}$ & Air & 56 & 39 & 1.9 \\
\hline 150 & $240 \min$ & Air & 60 & 36 & 2.2 \\
\hline 175 & $10 \mathrm{~min}$ & Air & 59 & 37 & 2.1 \\
\hline 175 & $240 \min$ & Air & 73 & 23 & 4.2 \\
\hline 200 & $10 \mathrm{~min}$ & Air & 69 & 26 & 3.5 \\
\hline 200 & $240 \min$ & Air & 81 & 16 & 6.8 \\
\hline 200 & $240 \min$ & DMF & 72 & 12 & 8.0 \\
\hline 250 & $10 \mathrm{~min}$ & Air & 80 & 20 & 5.3 \\
\hline 250 & $240 \min$ & DMF & 73 & 12 & 8.2 \\
\hline
\end{tabular}




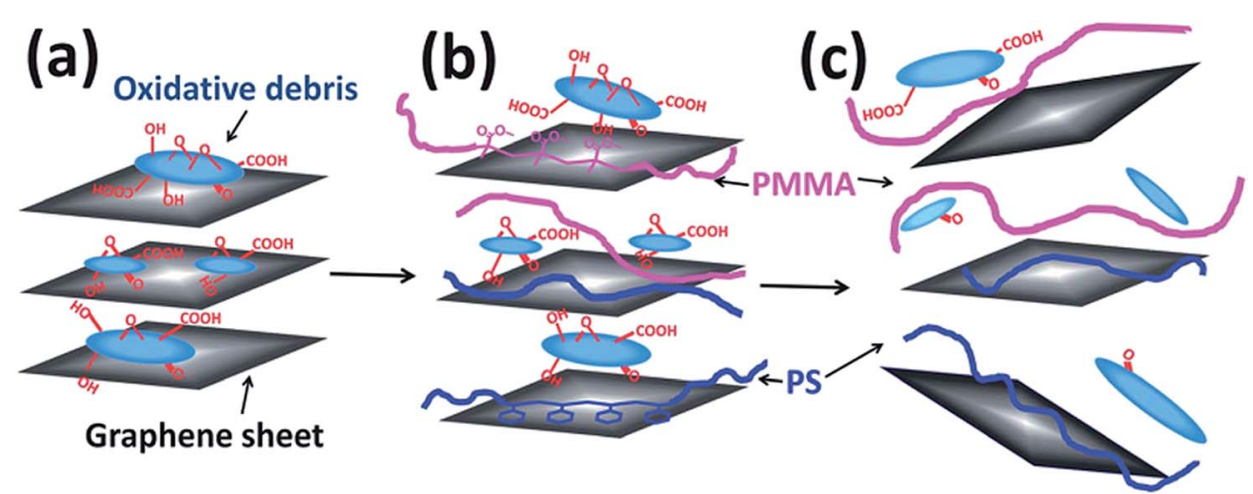

Fig. 8 Schematic of GO sheets model and its interaction with polymer (Ye et al. ${ }^{45}$ reproduced by the permission of The Royal Society of Chemistry).

polymer and the resistivity of the nanocomposites as a function of annealing time is necessary to understand the mechanism of thermal reduction of GO. Glover et al. ${ }^{44}$ studied the effect of various polymers with respect to the temperatures. The $\mathrm{C} / \mathrm{O}$ ratio in GO was monitored, as described in Table 2.

In addition, the polymer chemistry i.e. polarity and aromaticity dictates efficient reduction and the overall state of dispersion. The exact mechanism of reduction of GO is not yet known, which is due to lack of direct measurement of the reduction process and the chemical reactions that take place during processing in the melt. However, with the limited results, Ye et al..$^{45}$ arrived at some understanding. The decrease in the reducing temperatures in the presence of polymer is related to the interactions between GO sheets and the polymer matrix. Further, Rourke et al. $^{46}$ proposed that GO are large sheets of graphene with oxidative debris adhered to sheets by $\pi-\pi$ stacking or van der Waals interactions.

Fig. 8 illustrates schematically the GO sheets and its interaction with the polymer. From Fig. 8, it is clear that aromatic polymer such as polystyrene (PS) initially adsorb on the graphene sheets by $\pi-\pi$ stacking between the continuous phenyl rings and the conjugated basal planes of graphene. This adsorption facilitates intercalation of GO sheets. Further, the $\pi-\pi$ stacking counter balances the interactions between graphene sheets and oxidative debris, thus facilitating strong adhesion of polymer chains on the sheets. This adhesion will eliminate the oxidative debris from the surface of the sheet. Both the attachment of polymer chain and the elimination of oxidative debris leads to the reduction of GO thermally, and requires low energy and low reducing temperatures. It is envisaged that polar polymers, such as PMMA, forms hydrogen bonding with the oxidative debris. This further assists in peeling off the oxidative debris from the sheet. As a result, the reduction of GO in polar polymers require relatively less energy and reducing temperatures.

Apart from enhanced electrical conductivity during in situ reduction of $\mathrm{GO}$, observed to compatibilize immiscible poly(methyl methacrylate)/polystyrene (PMMA/PS, 80/20) blends. ${ }^{47}$

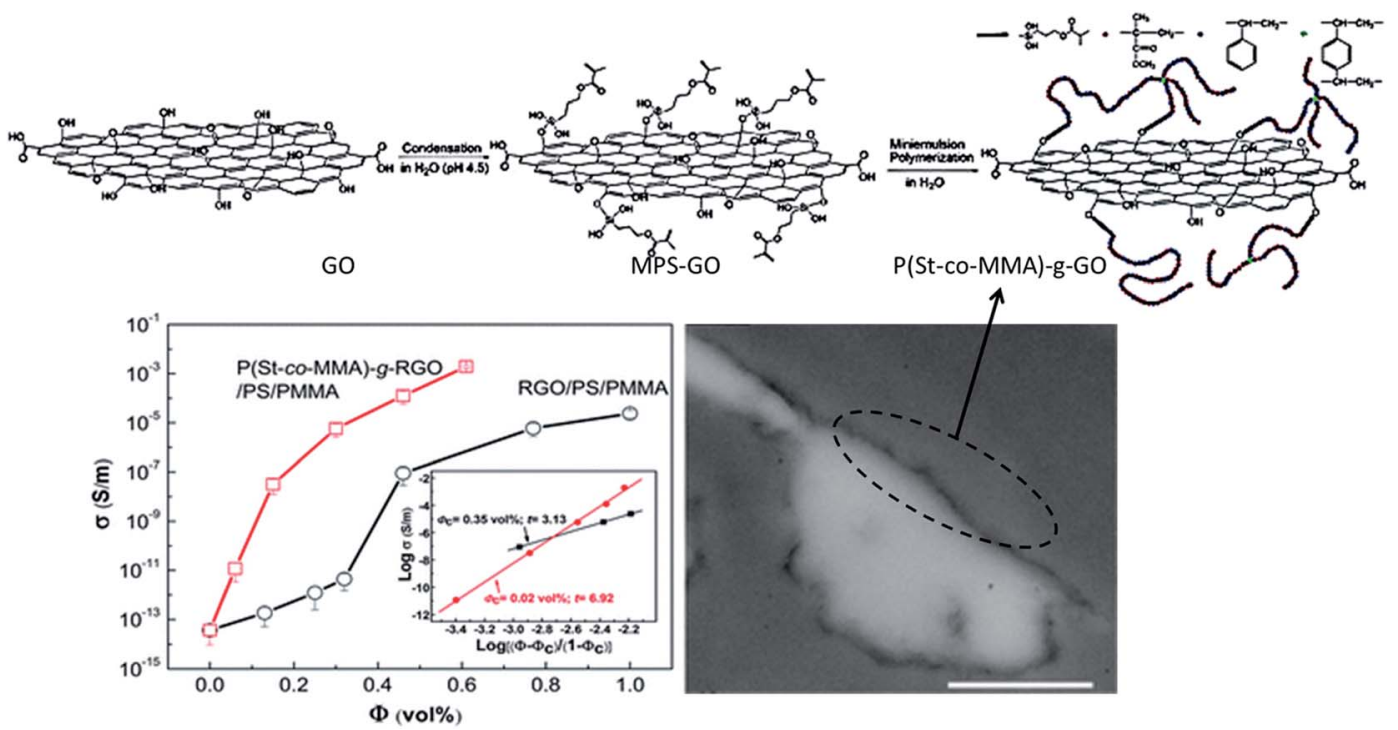

Fig. 9 Schematic of covalent functionalization of GO and conductivity plot of PS/PMMA blend with rGO and P(St-co-MMA) copolymer grafted $\mathrm{GO}$ (Tan et al. ${ }^{48}$ reproduced by the permission of The Royal Society of Chemistry). 

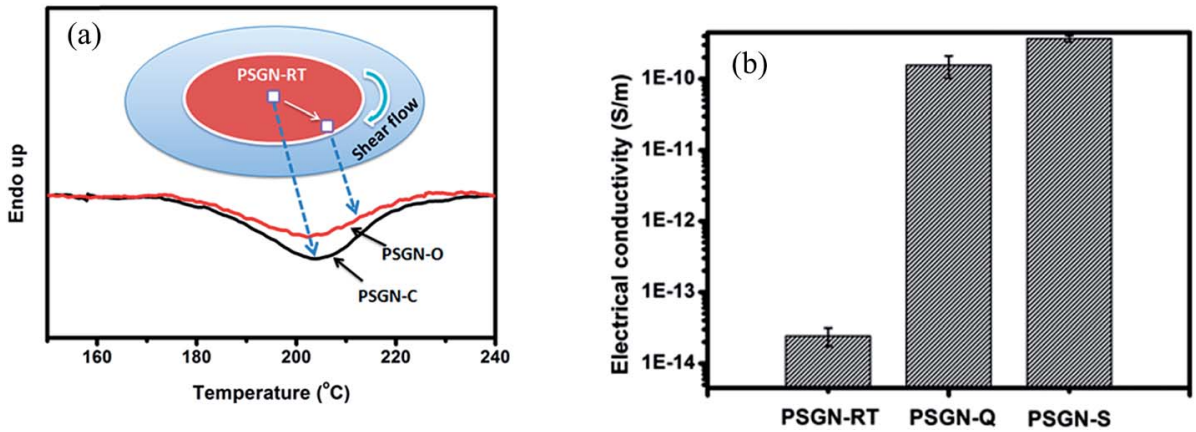

Fig. 10 Shows (a) schematic of DSC scans of GO in various zones (b) exhibits conductivity of neat polystyrene composite, quiescent and sheared polystyrene composite (Ye et al. ${ }^{45}$ reproduced by the permission of The Royal Society of Chemistry).

The droplet diameter of the dispersed phase (PS) phase significantly reduced in the presence of GO. During processing, at higher temperatures, the in situ thermal reduction of GO renders more hydrophobicity in GO and further suppresses the coalescence of PS droplets. However, the electrical conductivity of the samples were not reported in this study.

Recently, Tan et al. ${ }^{48}$ covalently functionalized GO with a copolymer and converted GO to reduced GO in situ during molding of PMMA/PS blends. This facile method resulted in localization of GO at the interface of PMMA/PS blends and further resulted in low percolation threshold of $0.02 \mathrm{vol} \%$. This is the lowest reported value in context to graphene as a nanofillers. Further, from Fig. 9, it is evident that the conductivity increased by 2 orders of magnitude for the composite containing $\mathrm{P}(\mathrm{St}-\mathrm{co}$-MMA) copolymer grafted GO. The TEM image indicated localization of GO sheets at the interface.

\section{Shear induced in situ reduction of GO}

From the above discussion, it is clear that temperature and external forces like compression strain can assist in the in situ reduction of GO. However, in practice, heat can be accompanied by shear forces for further reduction of GO. In this context, Ye et al. studied ${ }^{45}$ the in situ reduction of GO in quiescent melt and under shear. They demonstrated that the composites which were sheared in the melt had a higher degree of reduction as compared to quiescent condition. They observed that the

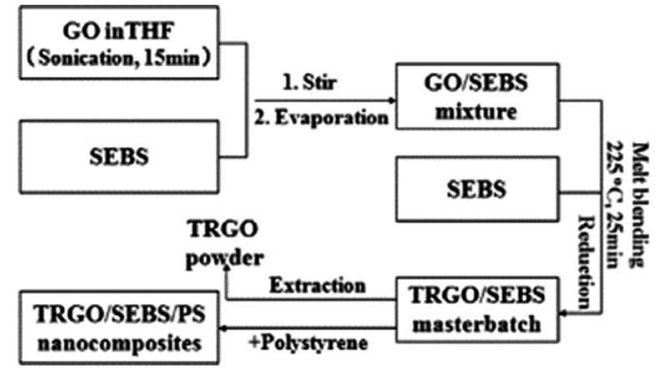

Fig. 11 In situ reduction of GO via master batch in polystyrene (You et al. ${ }^{49}$ reproduced by permission of John Wiley and Sons, Copyright $(\subset)$ 2014 Wiley Ltd. All rights reserved). reduction was quite high under low shear, which further confirms the effect of shear on reduction of GO. This efficient reduction is associated with enhanced $\pi-\pi$ stacking and the interaction between graphene sheets and the matrix. Further, they showed that an increase in conductivity of $c a$. 4 orders after shear. This was ascribed to significant reduction of GO during melt blending under high shear forces. Moreover, they observed that the central part of the composite sample (as shown in Fig. 10) exhibited higher reduction than the outer part by monitoring the enthalpy. This change in enthalpy was attributed to energy consumption required for the reduction of GO. As the outer part of the sample experiences higher shear force than the central part, the degree of reduction is higher at the center.

Another less explored strategy for in situ reduction of GO is the use of masterbatch. You et al..$^{49}$ prepared a master batch of graphene oxide with styrene-ethylene/butylene-styrene (SEBS) triblock copolymer by melt mixing at $225^{\circ} \mathrm{C}$ and they obtained a high degree of reduction. The resultant master batch was further diluted with polystyrene in the subsequent melt mixing process for improving mechanical properties. They reported three orders of magnitude increase in conductivity in reduced GO that was extracted from SEBS as shown schematically in Fig. 11.

\section{Conclusions and outlook}

Recent advances in in situ reduction of GO during the preparation of conducting polymeric nanocomposites has been reviewed here. The effect of presence of oxygen species on the

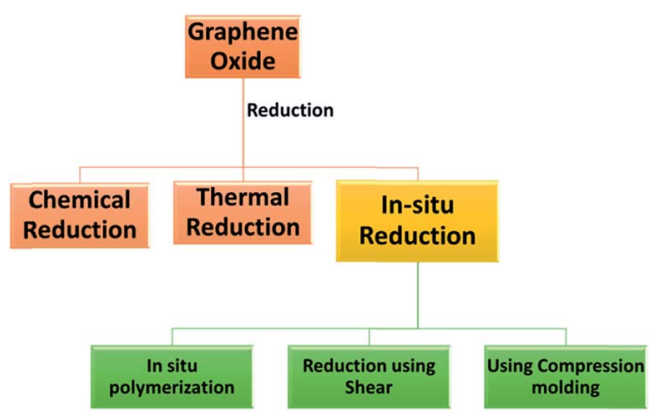

Fig. 12 Outline of various strategies to reduce GO 
Table 3 Various polymer composites involving in situ reduction of GO and their electrical conductivity ${ }^{39}$

\begin{tabular}{|c|c|c|c|c|}
\hline Matrix & $\begin{array}{l}\text { Reduced GO content } \\
\text { (vol\%) }\end{array}$ & Reduction method & $\begin{array}{l}\text { Conductivity } \\
\left(\mathrm{S} \mathrm{m}^{-1}\right)\end{array}$ & Ref. \\
\hline - & 100 & - & 750 & 39 \\
\hline UHMWPE & 0.66 & Compression molding & 3.4 & 39 \\
\hline PVDF & 0.75 & Compression molding & 0.02 & 50 \\
\hline PVDF & 1.70 & Solar electromagnetic radiation & 0.04 & 51 \\
\hline PVDF & 2.75 & Compression molding & 0.0002 & 37 \\
\hline PA6 & 1.07 & In situ polymerization & 0.028 & 52 \\
\hline PMMA & 2.79 & Compression molding & 0.94 & 45 \\
\hline SEBS & 2.13 & Compression molding & 0.34 & 45 \\
\hline PET & 17.4 & In situ polymerization & 0.56 & 29 \\
\hline
\end{tabular}

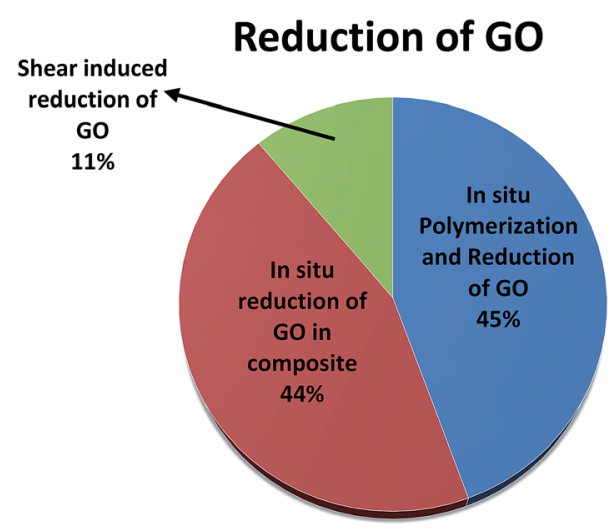

Fig. 13 Pie chart of various strategies to reduce GO in situ.

overall electrical properties of the nanocomposites is juxtaposed to highlight and compare the efficacy of the various strategies employed to reduce GO. Several strategies such as in situ polymerization, hot press, and shear are critically assessed here. In situ polymerization and simultaneous reduction of GO in polymer matrix, demonstrate homogeneous dispersion of reduced GO which results in lower electrical percolation threshold. From the existing literature, it is evident that hot press is not sufficient for complete reduction of GO but the efficacy of reduction can be enhanced many folds if coupled with shear forces. Among the different strategies, shear in combination with heat was observed to be the most effective strategy. This opens new avenues in designing GO based conducting nanocomposites. Fig. 12 outlines various strategies involving in situ reduction of GO and Table 3 highlights the electrical conductivities achieved by in situ reduction of GO. The effect of polymer chemistry on the in situ reduction of GO is scarce and need to be explored more. Fig. 13 shows a pie chart of various strategies adopted for in situ reduction of GO. It is interesting to note that in situ polymerization and simultaneous reduction of GO provide a homogeneous dispersion and reduce the electrical percolation threshold. Shear induced reduction of GO shows promising reduction of $\mathrm{GO}$ and this method needs to explored in detail in future.

From the existing literature concerning the reduction of GO it is apparent that in situ reduction of GO to design highly conducting polymer composites is a better way as compared to ex situ reduction processes which often involves harsh treatments. More emphasis should be given in this direction and more research should be pursued towards in situ reduction of GO during the composite preparation.

\section{References}

1 Y. Zhu, S. Murali, W. Cai, X. Li, J. W. Suk, J. R. Potts and R. S. Ruoff, Graphene and graphene oxide: synthesis, properties, and applications, Adv. Mater., 2010, 22, 39063924.

2 S. Pei and H.-M. Cheng, The reduction of graphene oxide, Carbon, 2012, 50, 3210-3228.

3 D. Boukhvalov and M. Katsnelson, Chemical functionalization of graphene, J. Phys.: Condens. Matter, 2009, 21, 344205.

4 Z. Yan, D. L. Nika and A. A. Balandin, Thermal properties of graphene and few-layer graphene: applications in electronics, IET Circuits, Devices \& Systems, 2015, 9, 4-12.

5 B. Luo, S. Liu and L. Zhi, Chemical Approaches toward Graphene-Based Nanomaterials and their Applications in Energy-Related Areas, Small, 2012, 8, 630-646.

6 G. Mittal, V. Dhand, K. Y. Rhee, S.-J. Park and W. R. Lee, A review on carbon nanotubes and graphene as fillers in reinforced polymer nanocomposites, J. Ind. Eng. Chem., 2015, 21, 11-25.

7 B. Li, W. Hou, J. Sun, S. Jiang, L. Xu, G. Li, M. A. Memon, J. Cao, Y. Huang, C. W. Bielawski and J. Geng, Tunable Functionalization of Graphene Oxide Sheets through Surface-Initiated Cationic Polymerization, Macromolecules, 2015, 48, 994-1001.

8 S. Kumar, S. Raj, E. Kolanthai, A. K. Sood, S. Sampath and K. Chatterjee, Chemical Functionalization of Graphene To Augment Stem Cell Osteogenesis and Inhibit Biofilm Formation on Polymer Composites for Orthopedic Applications, ACS Appl. Mater. Interfaces, 2015, 7, 3237-3252.

9 S. Kumar and K. Chatterjee, Strontium eluting graphene hybrid nanoparticles augment osteogenesis in a 3D tissue scaffold, Nanoscale, 2015, 7, 2023-2033.

10 T. Thanh Tung, M. Castro, J. Feller and T. Kim, in GrapheneBased Polymer Nanocomposites in Electronics, ed. K. K. Sadasivuni, D. Ponnamma, J. Kim and S. Thomas, Springer International Publishing, 2015, pp. 253-275. 
11 H. T. Xing, J. H. Chen, X. Sun, Y. H. Huang, Z. B. Su, S. R. Hu, W. Weng, S. X. Li, H. X. Guo, W. B. Wu, Y. S. He, F. M. Li and Y. Huang, $\mathrm{NH}_{2}$-rich polymer/graphene oxide use as a novel adsorbent for removal of $\mathrm{Cu}(\mathrm{ii})$ from aqueous solution, Chem. Eng. J., 2015, 263, 280-289.

12 S. Zhan, D. Zhu, S. Ma, W. Yu, Y. Jia, Y. Li, H. Yu and Z. Shen, Highly Efficient Removal of Pathogenic Bacteria with Magnetic Graphene Composite, ACS Appl. Mater. Interfaces, 2015, 7, 4290-4298.

13 D. Dinda and S. Kumar Saha, Sulfuric acid doped poly diaminopyridine/graphene composite to remove high concentration of toxic $\mathrm{Cr}(\mathrm{vI})$, J. Hazard. Mater., 2015, 291, 93-101.

14 S. Xia and M. Ni, Preparation of poly(vinylidene fluoride) membranes with graphene oxide addition for natural organic matter removal, J. Membr. Sci., 2015, 473, 54-62.

15 Q. Huang, J. Wang, W. Wei, Q. Yan, C. Wu and X. Zhu, A facile and green method for synthesis of reduced graphene oxide/Ag hybrids as efficient surface enhanced Raman scattering platforms, J. Hazard. Mater., 2015, 283, 123-130.

$16 \mathrm{~S}$. Wu, Z. Liu, N. Liu and Z. Ma, Oligomeric 2aminothiophenol decorated carboxyl graphene: a new surface enhanced Raman reporter and its application in immunosensing, Sens. Actuators, B, 2015, 206, 502-507.

17 D. R. Dreyer, S. Park, C. W. Bielawski and R. S. Ruoff, The chemistry of graphene oxide, Chem. Soc. Rev., 2010, 39, 228-240.

18 S. Chen, X. Li, Y. Zhao, L. Chang and J. Qi, Graphene oxide shell-isolated $\mathrm{Ag}$ nanoparticles for surface-enhanced Raman scattering, Carbon, 2015, 81, 767-772.

19 M. Segal, Selling graphene by the ton, Nat. Nanotechnol., 2009, 4, 612-614.

20 B. C. Brodie, On the Atomic Weight of Graphite, Philos. Trans. R. Soc. London, 1859, 149, 249-259.

21 L. Staudenmaier, Method for the preparation of graphitic acid, Ber. Dtsch. Chem. Ges., 1898, 31, 1481-1487.

22 W. S. Hummers and R. E. Offeman, Preparation of Graphitic Oxide, J. Am. Chem. Soc., 1958, 80, 1339.

23 S. Unarunotai, Y. Murata, C. E. Chialvo, N. Mason, I. Petrov, R. G. Nuzzo, J. S. Moore and J. A. Rogers, Conjugated carbon monolayer membranes: methods for synthesis and integration, Adv. Mater., 2010, 22, 1072-1077.

24 Y. Zhu, S. Murali, M. D. Stoller, A. Velamakanni, R. D. Piner and R. S. Ruoff, Microwave assisted exfoliation and reduction of graphite oxide for ultracapacitors, Carbon, 2010, 48, 2118-2122.

25 C. K. Chua and M. Pumera, Chemical reduction of graphene oxide: a synthetic chemistry viewpoint, Chem. Soc. Rev., 2014, 43, 291-312.

26 D. R. Dreyer, R. S. Ruoff and C. W. Bielawski, From conception to realization: an historial account of graphene and some perspectives for its future, Angew. Chem., Int. Ed., 2010, 49, 9336-9344.

27 W.-F. Ji, K.-C. Chang, M.-C. Lai, C.-W. Li, S.-C. Hsu, T.-L. Chuang, J.-M. Yeh and W.-R. Liu, Preparation and comparison of the physical properties of PMMA/thermally reduced graphene oxides composites with different carboxylic group content of thermally reduced graphene oxides, Composites, Part A, 2014, 65, 108-114.

28 J. R. Potts, D. R. Dreyer, C. W. Bielawski and R. S. Ruoff, Graphene-based polymer nanocomposites, Polymer, 2011, 52, 5-25.

29 K. Liu, L. Chen, Y. Chen, J. Wu, W. Zhang, F. Chen and Q. Fu, Preparation of polyester/reduced graphene oxide composites via in situ melt polycondensation and simultaneous thermoreduction of graphene oxide, J. Mater. Chem., 2011, 21, 86128617.

30 T.-X. Jin, C. Liu, M. Zhou, S.-g. Chai, F. Chen and Q. Fu, Crystallization, mechanical performance and hydrolytic degradation of poly(butylene succinate)/graphene oxide nanocomposites obtained via in situ polymerization, Composites, Part A, 2015, 68, 193-201.

31 F.-Y. Yuan, H.-B. Zhang, X. Li, H.-L. Ma, X.-Z. Li and Z.-Z. Yu, In situ chemical reduction and functionalization of graphene oxide for electrically conductive phenol formaldehyde composites, Carbon, 2014, 68, 653-661.

32 P. Ding, S. Su, N. Song, S. Tang, Y. Liu and L. Shi, Highly thermal conductive composites with polyamide- 6 covalently-grafted graphene by an in situ polymerization and thermal reduction process, Carbon, 2014, 66, 576-584.

33 J. Fan, R. Huang, S. Ye, T. Li and J. Feng, The probable influence of in situ thermal reduction of graphene oxides on the crystallization behavior of isotactic polypropylene, Polymer, 2014, 55, 4341-4347.

34 M. Fang, K. Wang, H. Lu, Y. Yang and S. Nutt, Single-layer graphene nanosheets with controlled grafting of polymer chains, J. Mater. Chem., 2010, 20, 1982-1992.

35 X. Qian, L. Song, B. Yu, W. Yang, B. Wang, Y. Hu and R. K. Yuen, One-pot surface functionalization and reduction of graphene oxide with long-chain molecules: preparation and its enhancement on the thermal and mechanical properties of polyurea, Chem. Eng. J., 2014, 236, 233-241.

36 M. Traina and A. Pegoretti, In situ reduction of graphene oxide dispersed in a polymer matrix, J. Nanopart. Res., 2012, 14, 1-6.

37 H. Tang, G. J. Ehlert, Y. Lin and H. A. Sodano, Highly efficient synthesis of graphene nanocomposites, Nano Lett., 2011, 12, 84-90.

38 L. He and S. C. Tjong, A graphene oxide-polyvinylidene fluoride mixture as a precursor for fabricating thermally reduced graphene oxide-polyvinylidene fluoride composites, RSC Adv., 2013, 3, 22981-22987.

39 Y. Ding-Xiang, P. Huan, X. Ling, B. Yu, R. Peng-Gang, L. Jun and L. Zhong-Ming, Electromagnetic interference shielding of segregated polymer composite with an ultralow loading of in situ thermally reduced graphene oxide, Nanotechnology, 2014, 25, 145705.

40 D.-X. Yan, H. Pang, B. Li, R. Vajtai, L. Xu, P.-G. Ren, J.-H. Wang and Z.-M. Li, Structured Reduced Graphene Oxide/Polymer Composites for Ultra-Efficient Electromagnetic Interference Shielding, Adv. Funct. Mater., 2015, 25, 559-566. 
41 Y. Shen, T. Jing, W. Ren, J. Zhang, Z.-G. Jiang, Z.-Z. Yu and A. Dasari, Chemical and thermal reduction of graphene oxide and its electrically conductive polylactic acid nanocomposites, Compos. Sci. Technol., 2012, 72, 1430-1435.

42 Y. Zhu, M. D. Stoller, W. Cai, A. Velamakanni, R. D. Piner, D. Chen and R. S. Ruoff, Exfoliation of graphite oxide in propylene carbonate and thermal reduction of the resulting graphene oxide platelets, ACS Nano, 2010, 4, 1227-1233.

43 W. Li, X.-Z. Tang, H.-B. Zhang, Z.-G. Jiang, Z.-Z. Yu, X.-S. Du and Y.-W. Mai, Simultaneous surface functionalization and reduction of graphene oxide with octadecylamine for electrically conductive polystyrene composites, Carbon, 2011, 49, 4724-4730.

44 A. J. Glover, M. Cai, K. R. Overdeep, D. E. Kranbuehl and H. C. Schniepp, In situ reduction of graphene oxide in polymers, Macromolecules, 2011, 44, 9821-9829.

$45 \mathrm{~S}$. Ye and J. Feng, A new insight into the in situ thermal reduction of graphene oxide dispersed in a polymer matrix, Polym. Chem., 2013, 4, 1765-1768.

46 J. P. Rourke, P. A. Pandey, J. J. Moore, M. Bates, I. A. Kinloch, R. J. Young and N. R. Wilson, The Real Graphene Oxide Revealed: Stripping the Oxidative Debris from the Graphene-like Sheets, Angew. Chem., Int. Ed., 2011, 50, 3173-3177.
47 S. Ye, Y. Cao, J. Feng and P. Wu, Temperature-dependent compatibilizing effect of graphene oxide as a compatibilizer for immiscible polymer blends, RSC Adv., 2013, 3, 7987-7995.

48 Y. Tan, L. Fang, J. Xiao, Y. Song and Q. Zheng, Grafting of copolymers onto graphene by miniemulsion polymerization for conductive polymer composites: improved electrical conductivity and compatibility induced by interfacial distribution of graphene, Polym. Chem., 2013, 4, 2939-2944.

49 F. You, D. Wang, J. Cao, X. Li, Z. M. Dang and G. H. Hu, In situ thermal reduction of graphene oxide in a styreneethylene/butylene-styrene triblock copolymer via melt blending, Polym. Int., 2014, 63, 93-99.

50 M. Li, C. Gao, H. Hu and Z. Zhao, Electrical conductivity of thermally reduced graphene oxide/polymer composites with a segregated structure, Carbon, 2013, 65, 371-373.

51 V. Eswaraiah, K. Balasubramaniam and S. Ramaprabhu, One-pot synthesis of conducting graphene-polymer composites and their strain sensing application, Nanoscale, 2012, 4, 1258-1262.

52 D. Zheng, G. Tang, H.-B. Zhang, Z.-Z. Yu, F. Yavari, N. Koratkar, S.-H. Lim and M.-W. Lee, In situ thermal reduction of graphene oxide for high electrical conductivity and low percolation threshold in polyamide 6 nanocomposites, Compos. Sci. Technol., 2012, 72, 284-289. 